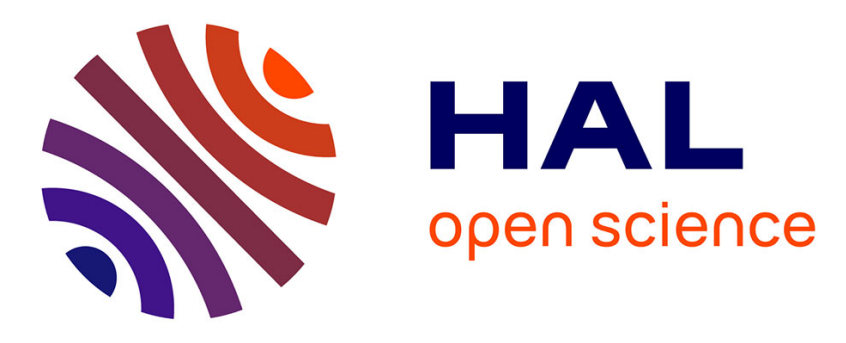

\title{
Exhaustive Modal Analysis of Interconnected European Power System
}

\author{
Mohamed Kouki, Bogdan Marinescu, Florent Xavier
}

\section{To cite this version:}

Mohamed Kouki, Bogdan Marinescu, Florent Xavier. Exhaustive Modal Analysis of Interconnected European Power System: Exhaustive Modal Analysis of Interconnected European Power System. 2020 IEEE Electric Power and Energy Conference (EPEC), Nov 2020, Edmonton, AB, Canada. hal03103860

\section{HAL Id: hal-03103860 https://hal.science/hal-03103860}

Submitted on 8 Jan 2021

HAL is a multi-disciplinary open access archive for the deposit and dissemination of scientific research documents, whether they are published or not. The documents may come from teaching and research institutions in France or abroad, or from public or private research centers.
L'archive ouverte pluridisciplinaire HAL, est destinée au dépôt et à la diffusion de documents scientifiques de niveau recherche, publiés ou non, émanant des établissements d'enseignement et de recherche français ou étrangers, des laboratoires publics ou privés. 


\section{Exhaustive Modal Analysis of Interconnected European Power System}

\author{
Mohamed Kouki \& Bogdan Marinescu \\ Ecole Centrale Nantes-LS2N \\ 1 , rue de la Nö̈ \\ 44321 Nantes Cedex 3-France \\ \{Mohamed.Kouki, Bogdan.Marinescu\}@ec-nantes.fr
}

\author{
Florent Xavier \\ $R T E-R \& D$ \\ Immeuble WINDOW-7C, Place du Dôme \\ 92073 Paris La Défense Cedex-France \\ florent.xavier@rte-france.com
}

\begin{abstract}
The interconnected European power system is evolving due to the new extensions and to the systematic integration of Power Park Modules, i.e., unit or ensemble of units generation electricity (Renewable Energy (RE), Distributed Generation (DG), etc), which is connected to the grid by power electronics and FACTS (STATCOM, HVDC,...). As a consequence, the increase of the size and the emergence of the new oscillatory modes which involve distant dynamic devices, i.e., of coupling modes (modes associated to generator/turbine inertia, and modes associated to Power Park Modules), and of modes related to various regulations. In this paper, a full scan of European power system is given. An exhaustive method that determines all oscillatory modes independently of the system's order, of the topology and without a priori knowledge about the system, is used. The exhaustive method is fully analytic. Indeed, it is based only on the state-space realisation of any given power system and does not require operator manipulation like dynamic simulations. To this end, we quantify and analyse the interaction between the different inputs/outputs of a given system. This leads to the identification of classes of coupled dynamic devices of the power system. Next, starting from a reduced number of classes, a selective modal analysis method is applied to each class to put into evidence a good approximation of all oscillatory modes. Finally, a complete classification of the oscillatory modes found at the previous stage is provided in terms of the types (interarea, electrical, ...) and their characteristics (oscillation frequency, damping, participation, ...). 2900 oscillatory modes were found for the studied model of the European system.

Index Terms-coupling/inter-area modes, European power system, dynamic reference model, large-scale systems, modal analysis.
\end{abstract}

\section{INTRODUCTION}

During the last years, interconnected European power system has evolved. First, its size has increased, due to the new extensions like, e.g, interconnection with the Turkish power system. Next, its structure changed also due to systematic insertion of Power Park Modules (PPMs) for the renewable sources of for the HVDCs systematically used to reinforce border interconnections (e.g., Spain-France or France-Italy). Therefore, the interconnected dynamic devices are not only the power plants with synchronous machines, but they also contain the PPMs. The eigenanalysis of the resulting mathematical model is becoming very difficult, especially when the oscillatory dynamics are expected in an exhaustive manner, i.e., a full scan of the system has to be done and computation of only some preselected modes it is not enaugh. In fact, an exhaustive analysis should be able to determine all oscillatory modes, not only the inter-area modes, but also the electrical modes (modes related to the electrical parts of distant classic generators and the ones related to the PPMs). For example, In [1], [2], [6] and [7] coupling modes associated to electrical part of the distant calssic synchrounous generators are highlighted but not of inter-area nature. In [8], and [7] oscillatory modes associated to converters of distant HVDCs, PVs and winds were also highlighted and analysed. Therefore, all these oscillatory modes will be called coupling modes in the sequel.

Several approaches of modal analysis that aim to find all coupling modes of large scale power systems have been developed and discussed (see, e.g., [3]). They are often non exhaustive and provide only a selective modal analysis. This means that, if good initial guesses are given, only few modes can be found in an iterative manner. This supposes that some knowledge about the system is available which is not always the case.

To overcome these difficulties, two exhaustive methodologies were discussed and developed in our previous works [6], [7]. In fact, they provide a way to systematically excite and put into evidence all the coupling modes of a given power system. In addition, they quantify the interactions between the different dynamic devices of any given power system. Indeed, for each device, one can find the other devices to which it is coupled. These coupled devices should be aggregated into a class, and for all devices a set of classes can be constructed. These latter should be used in computing of the coupling modes based on a specific selective modal analysis method [3], [9].

To put into evidence all the European power system modes, the method proposed in [7] is used here with some modifications for large-scale use. Importantly, the analysis of the European power system or of a system of comparable size and complexity in exhaustive way is a novelty in modal analysis and has never been addressed before. Indeed, not only the well-known inter-area modes can be found, but all the coupling modes are put into evidence. In addition, determining the low and high frequencies modes of the network becomes feasible. 
As well, aggregating the dynamic devices according to the nature of the coupling modes or according to the oscillation frequencies it is also possible. Preliminary modal analysis results of the European power sysem were presented in [7].

The analysis is carried out on the Dynamic Reference Model (DRM), which was developed for the IPS/UPS EC study and next maintained [11]. The rest of this paper is organized as follows. Section II provides the mathematical model associated to power system and a brief description of the exhaustive modal analysis methods that were developed and discussed in [6] and [7]. Section III details the specific implementation of the analytic method to large-scale systems. The results of the full modal analysis of the European power system are reported in Section IV. Conclusions are given in Section V.

\section{BASIC TOOLS}

In this section we present the mathematical model used for power system and a brief description of exhaustive modal analysis methodologies developed in [6] and [7], which will be used subsequently.

\section{A. Power system description}

A power system is often modeled by a set of Differential and Algebraic Equations (DAE) called DAE form

$$
\left\{\begin{array}{c}
\dot{x}=f(x(t), w(t)) \\
0=g(x(t), w(t)),
\end{array}\right.
$$

where, $x(t)$ and $w(t)$ are the vectors of differential and algebraic variables, respectively. $x$ represents the state variables (machines speeds, regulators, active power,...), while $w$ mainly contains the bus voltages of the power system.

Linearization of (1) around an equilibrium point $\left(x_{0}, w_{0}\right)$ gives

$$
\dot{x}=A x,
$$

where $A \in \mathbb{R}^{n * n}$.

If a vector of input variables $u$ and a vector of output variables $y$ are also considered, (2) leads to an input-output linear model

$$
\left\{\begin{array}{l}
\dot{x}=A x+B u \\
y=C x
\end{array}\right.
$$

where, $B \in \mathbb{R}^{n * q}$ is the input matrix, and $C \in \mathbb{R}^{l * n}$ is the output matrix.

\section{B. New exhaustive modal analysis methodology}

[6] and [7] proposed an exhaustive modal analysis methods which hold for any type of coupling modes and power systems, in particular those with high power electronics penetration. They are structured into two main parts.

First, the identification of classes of the coupled dynamic devices (synchronous generators and converters) is done using the simulation technique [6] (i.e., exciting, one by one, each dynamic device with well-chosen events (such as shortcircuit)), or by analytic technique [7] (i.e., using the relative sensitivity technique to quantify the interaction between the inputs/outputs), and by analysing the correlation between the obtained outputs/sensitivities of all dynamic devices.

Next, the Selective Modal Analysis (SMA) method [9] is applied to each class in order to determine a good approximation of coupling modes.

SMA is an efficient approach for simplifying and analyzing a selected part of a large-scale linear system (2). It is based on the split of the state vector $x$ into two parts, a relevant part $r$ and a less relevant part $z$

$$
\dot{x}=\left[\begin{array}{l}
\dot{r} \\
\dot{z}
\end{array}\right]=\left[\begin{array}{ll}
A_{r r} & A_{r z} \\
A_{z r} & A_{z z}
\end{array}\right]\left[\begin{array}{l}
r \\
z
\end{array}\right] .
$$

This partition is illustrated on the Fig. 1.a. Based on this latter, the transfer function $H(s)$ of the less relevant dynamics could be generated.

$$
H(s)=A_{r z}\left(s I-A_{z z}\right)^{-1} A_{z r} .
$$

The eigencomputation is run only on the relevant dynamics

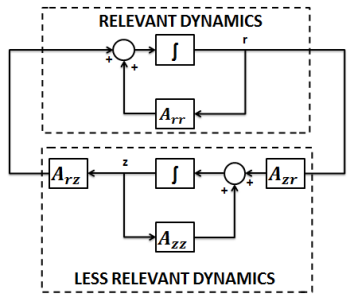

(a)

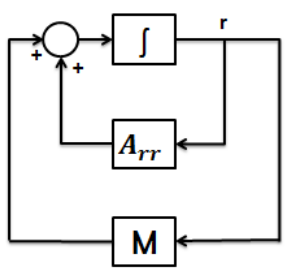

(b)
Fig. 1: The relevant and less relevant dynamics

by taking into account the small effect of the less relevant ones on the eigenvalues of interest. The less relevant dynamics can be exactly introduced in the relevant dynamics by a constant matrix $M$ that satisfies the linear system of (6).

$$
\mathbf{M} V_{r}=\left[H\left(\lambda_{1}\right) v_{1} \ldots H\left(\lambda_{r}\right) v_{r}\right]
$$

with $\lambda_{r}$ are the interest eigenvalues, and $V_{r}=\left[v_{1} \ldots v_{r}\right]$ is the matrix of the right eigenvectors associated to $\lambda_{r}$. The partition of Fig. 1.a could be represented now as shown in Fig. 1.b.

Notice that, in our case, the relevant part is composed by the states of dynamic devices selected in each class.

The flowchart (see Fig. 2) summarizes the main steps of the exhaustive approaches [6], [7].

This methodology is now improved to apply to large-scale power systems such as the European one.

\section{Exhaustive Modal AnAlysis OF MODERN LARGE-SCALE POWER Systems}

\section{A. Description of the European case}

We use for the European power system, the Dynamic Reference Model (DRM) which was developed for the IPS/UPS EC study and next maintained and currently used for stability studies [11]. It is a detailed nonlinear model including generators along with their regulations (AVRs, PSS and Governors). It consists of 1074 machines, 7652 buses, 10465 lines and 2550 transformers. The order of the resulting linear model, i.e., $n$ in (2), is 16784 . 


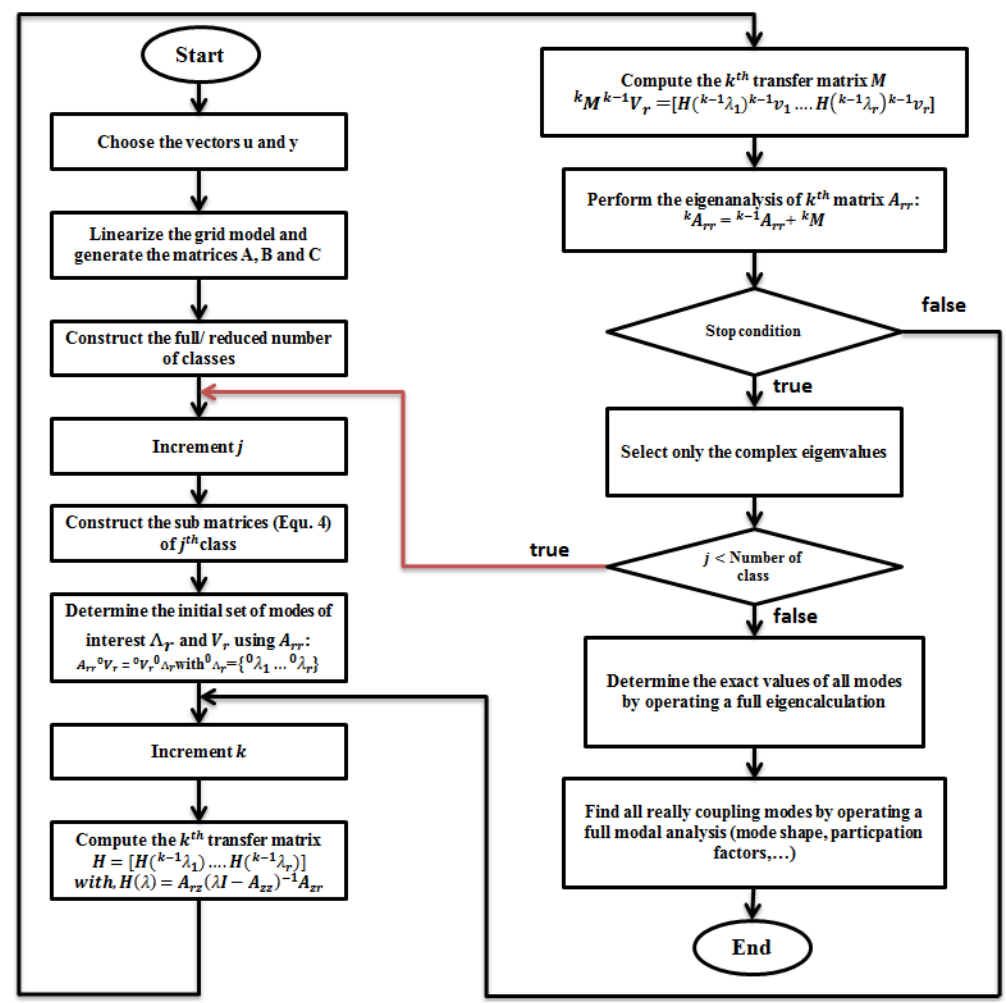

Fig. 2: Flowchart of the exhaustive modal analysis approach

\section{B. Specific large-scale implementation of the proposed method for exhaustive modal analysis}

1) Classes identification: In large-scale systyems, the quantification of the interactions and the construction of classes using the simulation technique are numerically fastidious. To overcome this problem, an analytic technique, called Relative Sensitivity Technique (RST) [4], [7], [10] is used here since it is applicable to large-scale systems such as the European one. The RST does not require operator manipulations, such as dynamic simulations, to identify the coupling classes.

In this work, the RST provides a matrix called, in the sequel, Relative Sensitivity Matrix (RSM) [7]. Based on the absolute value of $R S M$ and according to a chosen selection criterion $\delta$, the construction of the classes of coupled dynamic devices of a given power system is done:

- $R S M(i, j) \leq \delta \Rightarrow$ device $i$ and device $j$ are not coupled,

- $R S M(i, j)>\delta \Rightarrow$ device $i$ and device $j$ are coupled.

This protocol is applied on all the $R S M$ elements. Hence, a quasi-symmetric matrix made up with all coupled devices is obtained. Each row of $R S M$ corresponds to a class.

Notice that, the chosen inputs $u$ and outputs are respectively the references of the voltage regulators, and the speeds of synchronous generators.

2) Modes computing: The use of all obtained classes to put into evidence all coupling modes of large-scale power system gives a high redundancy between the computed modes and it is numerically fastidious. Instead, only some classes can be used. To identify them, a selection threshold $\sigma$ is used as follows:

- if the similarity (i.e., percentage of identic dynamic devices of two classes) between class $_{i}$ and class $_{j} \geq \sigma$ and $\operatorname{size}\left(\right.$ class $\left._{i}\right)>\operatorname{size}\left(\right.$ class $\left._{j}\right)$ then class $_{i}$ is one of the selected classes.

- if the similarity between class $_{i}$ and class $_{j}<\sigma$ then class $_{i}$ and class $_{j}$ are both selected.

Furthemore, in order to improve the computational efficiency of the specific implementation of the exhaustive approach, the stop condition used in [6], [7] is replaced here by a fixed number of iterations $k$.

\section{ANALysis RESUlts FOR THE EUROPEAN POWER SYSTEM}

\section{A. Classes and modes}

As mentioned above, the number of dynamic devices of European power system is equal to 1074 . Therefore, using the RST approximation and according to a selection criterion $\delta=0.001,1074$ classes are constructed.

As indicated in Table I, according to a selection criterion $\sigma=50 \%$, the number of final classes is 66 and the size of the largest class is 341 machines.

Notice that, the number of coupled machines of the largest class is a little large compared to the total number of machines. This is due to the small value chosen for the selection criterion $\delta$. In general, $\delta$ should be small in order to ensure high redundancy in the construction of classes. Therefore, a 
TABLE I: Coupled Classes of European power system

\begin{tabular}{|c|c|c|c|c|c|}
\hline Sys. & $\begin{array}{c}\text { Numb. } \\
\text { Mac. }\end{array}$ & $\begin{array}{c}\text { Numb. } \\
\text { Inp./Out. }\end{array}$ & Tot. Numb. Clas. & Red. Numb. Clas. & Size Larg. Clas. \\
\hline DRM & 1074 & $1074 / 1074$ & 1074 & 66 & 341 \\
\hline
\end{tabular}

TABLE II: Comparison between RST method and full model

\begin{tabular}{|c|c|c|c|c|c|}
\hline Number & Mode of full model & Mode with RST & Freq.(Hz) & Damp. & Type \\
\hline 1 & $-0.1247 \pm \mathrm{j}^{*} 1.1142$ & $-0.2362 \pm \mathrm{j}^{*} 1.1142$ & 0.1773 & 11.12 & ROT \\
\hline 2 & $-0.1980 \pm \mathrm{j}^{*} 1.5467$ & $-0.2003 \pm \mathrm{j}^{*} 2.0038$ & 0.2462 & 12.69 & ROT \\
\hline 3 & $-0.1489 \pm \mathrm{j}^{*} 2.0089$ & $-0.1315 \pm \mathrm{j}^{*} 2.0102$ & 0.3197 & 7.39 & ROT \\
\hline$\vdots$ & $\vdots$ & $\vdots$ & $\vdots$ & $\vdots$ & $\vdots$ \\
\hline 5 & $\vdots$ & $\vdots$ & $\vdots$ & $\vdots$ & $\vdots$ \\
\hline 6 & $-0.9241 \pm \mathrm{j}^{*} 2.5112$ & $-0.2445 \pm \mathrm{j}^{*} 2.3463$ & 0.39978 & 8.89 & ROT \\
\hline$\vdots$ & $\vdots$ & $-0.8531+\mathrm{j}^{*} 5.3899$ & 0.8529 & 16.88 & ROT \\
\hline 2198 & $-3.8818 \pm \mathrm{j}^{*} 1.2878$ & $-3.8836 \pm \mathrm{j}^{*} 1.2898$ & 0.2050 & 94.91 & SMQ \\
\hline$\vdots$ & $\vdots$ & $\vdots$ & $\vdots$ & $\vdots$ & $\vdots$ \\
\hline 2308 & $-0.1943 \pm \mathrm{j}^{*} 0.2883$ & $-0.1946 \pm \mathrm{j}^{*} 0.2832$ & 0.0459 & 55.88 & SMD \\
\hline$\vdots$ & $\vdots$ & $\vdots$ & $\vdots$ & $\vdots$ & $\vdots$ \\
\hline 2796 & $-1.1596 \pm \mathrm{j}^{*} 1.2160$ & $-1.1167 \pm \mathrm{j}^{*} 1.2311$ & 0.1935 & 69.01 & GOV \\
\hline$\vdots$ & $\vdots$ & $\vdots$ & $\vdots$ & $\vdots$ & $\vdots$ \\
\hline 2900 & $-120.4578 \pm \mathrm{j}^{*} 98.3070$ & $-120.3100 \pm \mathrm{j}^{*} 98.285$ & 15.6462 & 77.47 & EXC \\
\hline
\end{tabular}

maximum number of modes will be found for each class and, by the end, the eigencalculation is surely exhaustive in the sense that all the coupling modes of the studied power system will be found.

Starting from the final set of classes (66 classes) found for the European power system, SMA is applied on each class in order to put into evidence all coupling modes. The analysis of 66 classes provides real and complex modes. Here, only the complex modes are taken into account. Indeed, only the complex modes are associated to oscillatory phenomena. Furthermore, based on the complex modes, all coupling modes of the European power system can be found. Importantly, this is a challenge and a novelty in modal analysis of large-scale systems.

Table II provides all complex modes (2900 modes) found for the European power system. Their oscillation frequencies are up to $15.64 \mathrm{~Hz}$ and can be classified as reported in Table III.

In order to prove that the obtained modes are the exact values of the overall system, the classic selective modal analysis method, Modified Arnoldi (MA), was used. The modes generated by the SMA are used as initial guesses of MA iterations which converge to the exact values of the modes of the overall system. Notice that, $80 \%$ of 5800 complex modes are very precisely generated at the SMA stage and $20 \%$ are approximated with very small differences. This means that the selected classes (66 class) are the significant and efficient ones in the sense that there are no important interaction between the dynamics of each class and the rest of the system.

\section{B. Modes classification}

The complex modes found are classified according to their natures, oscillation frequencies, damping ratios and participation factors.

1) Classification according to the nature: The 2900 complex modes indicated in Table II are of 3 main types as reported in Table III: Electrical Modes (EM), Electromechanical Modes (EMM) i.e., inter-area and local modes, and Regulation

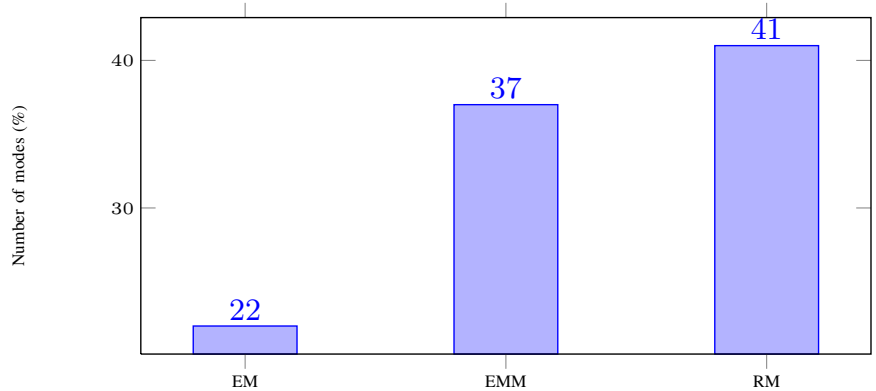

Fig. 3: Nature classification

Modes (RM). Their proportions are illustrated in Fig. 3. Notice that:

TABLE III: Base-Type of oscillatory modes

\begin{tabular}{|c|c|c|c|}
\hline DRM & EMM & EM & RM \\
\hline Numb. Mod. & 1091 & 636 & 1173 \\
\hline Freq. Max. (Hz) & 3.3985 & 5.6660 & 15.6462 \\
\hline
\end{tabular}

- an EM mode signifies that the most participation factors of its involved dynamic devices are associated to electrical parts (D-axes, or Q-axes, or injectors).

- an EMM mode means that the most participation factors of its involved dynamic devices are associated to rotor parts.

- an RM mode means that the most participation factors of its involved dynamic devices are associated to controller parts (Governor, AVR, PSS,...).

The first three modes of Table II are electromechanical of interarea nature. They are identified in the low frequency range $0.17 \mathrm{~Hz}$ to $0.30 \mathrm{~Hz}$.

The slowest mode of the European power system is the mode number 1 of Table II. The oscillation frequency of this mode is $0.17 \mathrm{~Hz}$. As indicated in columns 2 and 5 of Table IV, mode 1 involves 585 geographically distant synchronous generators with the highest participations associated to the rotors. The generators in Spain, Portugal and France swing against the generators in Turkey and Bulgaria. Therefore, this mode is an inter-area one and is one of the well-known modes identified in the studies of European power system performed by TSOs [5].

An example of electrical coupling mode is reported in Table $\mathrm{V}$. This mode involves 11 geographically distant synchronous generators with significant participation factors. The highest participation parts are not associated to the rotors but to Daxes. Therefore, this mode is not an inter-area mode but an electrical coupling mode. Furthermore, Fig. 4 illustrates the responses (ID current and IQ current) of most participating generators (G1, G2 and G11) to a short-circuit at the connection point of generator $G 1$. This short-circuit is applied at $t 1=60 \mathrm{~s}$ and eliminated at $t 2=60.1 \mathrm{~s}$. From this figure, notice an important variation at $t=60$ associated to the ID-current wich comes as consequence of electrical coupling. Table VI illustrates the results for mode number 2198 of Table 
TABLE IV: Machines with highest participation in mode 1

\begin{tabular}{|c|c|c|c|c|c|}
\hline Country & Mac. & Rel. Part. (\%) & Phase r. evec. $\delta$ (deg) & Rot. part & D-axes part \\
\hline Bulgaria & G1 & 95.9 & -5.4 & 0.2628 & 0.0082 \\
\hline Turkey & G2 & 100.0 & -1.0 & 01002 & 0.0025 \\
\hline Turkey & G3 & 100.0 & -1.0 & 0.0999 & 0.0024 \\
\hline Bulgaria & G4 & 55.3 & -0.8 & 0.0105 & 0.0001 \\
\hline Bulgaria & G5 & 94.0 & 4.0 & 0.0826 & 0.0611 \\
\hline Spain & G581 & 23.2 & 164.4 & 0.0035 & 0.0002 \\
\hline Portugal & G582 & 24.4 & 165.9 & 0.0101 & 0.0019 \\
\hline France & G583 & 34.4 & 175.6 & 0.0077 & 0.0001 \\
\hline Spain & G584 & 44.2 & 177.3 & 0.0127 & 0.0007 \\
\hline France & G585 & 36.8 & 178.9 & 0.0648 & 0.0040 \\
\hline
\end{tabular}

TABLE V: Machines with highest participation in mode 2308

\begin{tabular}{|c|c|c|c|c|c|}
\hline Country & Mac. & Rel. Part. (\%) & Phase r. evec. $\delta$ (deg) & Rot. part & D-axes part. \\
\hline \multirow{8}{*}{ Spain } & G1 & 100 & 0.0 & $0.5251 e^{-2}$ & 0.7833 \\
\hline & G2 & 15.4 & 69.6 & $0.6263 e^{-3}$ & 0.1239 \\
\hline & G3 & 3.5 & 42.5 & $0.8938 e^{-4}$ & $0.2660 e^{-1}$ \\
\hline & · & & & & \\
\hline & . & & & & \\
\hline & G9 & 1.8 & 162.5 & $0.6028 e^{-4}$ & $0.1456 e^{-1}$ \\
\hline & G10 & 13.4 & 103.0 & $0.4318 e^{-3}$ & 0.1085 \\
\hline & G11 & 20.2 & 103.0 & $0.6119 e^{-3}$ & 0.1582 \\
\hline
\end{tabular}

II. This latter is another electrical coupling mode type since it involves 3 distant generators with significant participation parts associated to the Q-axes as given in columns 6 and 8 of Table VI. The last type of oscillatory modes are the RM ones. They are local modes in which state variables of the controllers are dominant.

2) Classification according to the participation factor: Pariticipation factors give an interesting quantification of the spread of the modes, i.e., of how many distant devices they involve. Such classification is given in Fig. 5 for the electromechanical modes. Notice that, participations provided here are relative ones, i.e., purcentages of participation of the dominant device (which has 100\%). As a consequence, the group of 197 modes in Fig. 5 are of local nature, i.e., they mainly concern only one dynamic device. The second and third groups in Fig. 5 contain thus the inter-area modes of the system. Notice first that the inter-area modes are majoritary in the EMM class. The inter-area modes usually studied are the ones geographically spread and which involve a large number of machines, as for example, the East-West modes (see also link with the classification according to frequency). Other examples from the analysis done here:

- Mode number 5 in Table II has frequency $0.3997 \mathrm{~Hz}$ and involves 39 generators with a significant participation. These generators form two coherent groups: 6 generators in Denmark and Turkey (group 1) are in phase opposition to 33 generators in France, Poland, Greece, Bulgaria and Romania.

- Mode number 6 in Table II has frequency $0.8529 H z$ and involves 20 generators: 9 generators in Italy, France,

TABLE VI: Machines with highest participation in mode 2198

\begin{tabular}{|c|l|c|c|c|c|c|}
\hline Country & Mac. & Rel. Part. (\%) & Phase r. evec. $\delta$ (deg) & Rot. part & D-axes part. & Q-axes part. \\
\hline & G1000 & 100 & 0.0 & 0.1068 & 0.1850 & 0.3793 \\
POLAND & G1001 & 1.6 & -80.6 & 0.0245 & 0.0159 & 0.0298 \\
& G1003 & 2.2 & 147.5 & 0.0010 & 0.0044 & 0.0089 \\
\hline
\end{tabular}

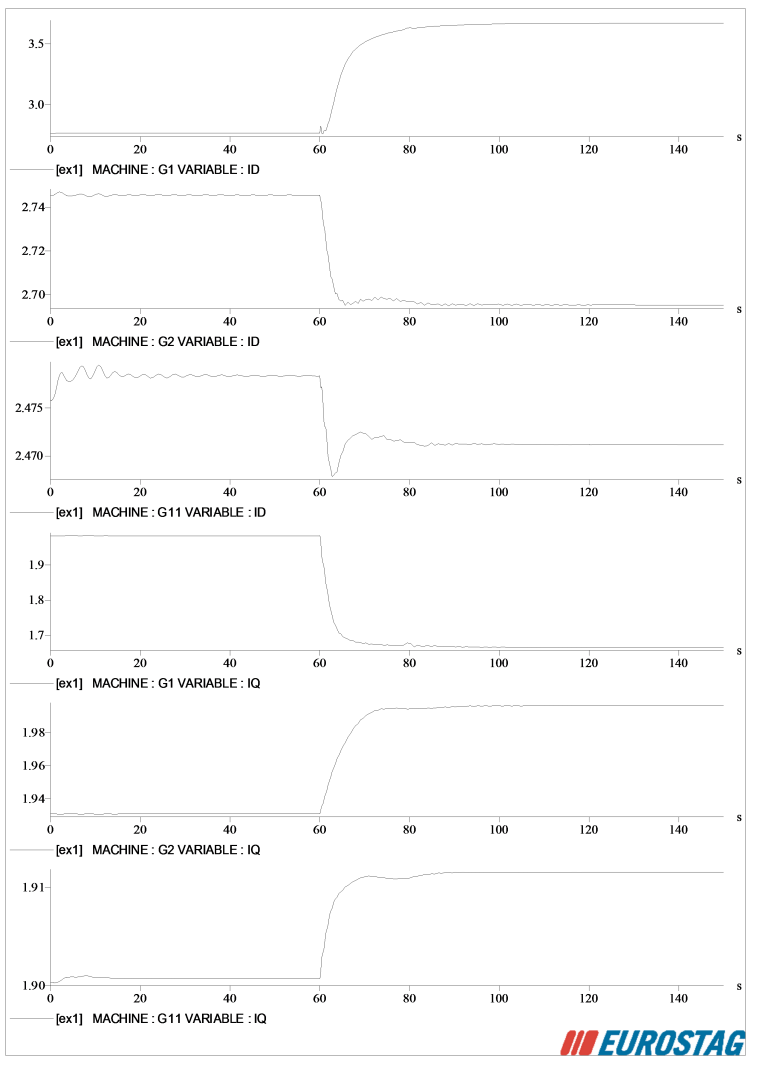

Fig. 4: Terminal current responses to the G1, G2 and G11

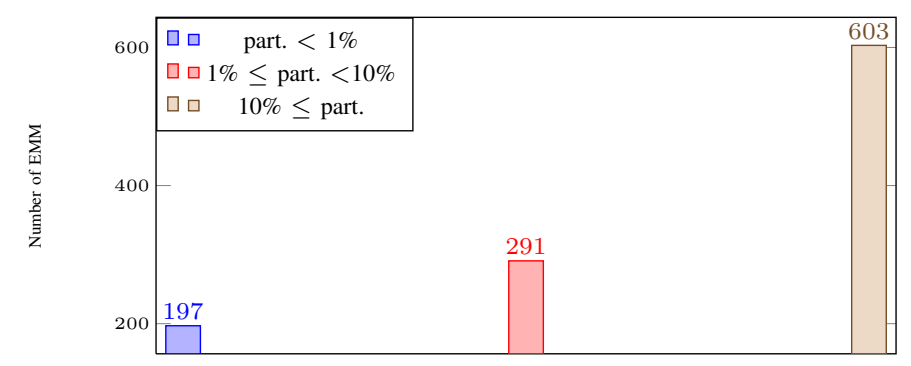

Fig. 5: Participation factor classification

Germany and Swizerland oscillate against 11 generators in Denmark and Czech Republic.

Notice however that, as shown in Fig. 6, most of the interarea modes are less spread modes since they involve a smaller number of machines (less than 18) with significant participation in the mode (more than 10\%). This is an important fact for stability analysis. From the control point of view, they can be damped in a more local manner, via classic PSS or POD installed on power converters (as for HVDCs, for example) in a more restricted area.

3) Classification according to the frequency: Classification of the oscillatory modes is done here according to the oscillation range of the frequency as illustrated in Fig. 7. Notice that majority of the electromechanical modes are of high frequencies (982 compared to only 15 modes in the usual 


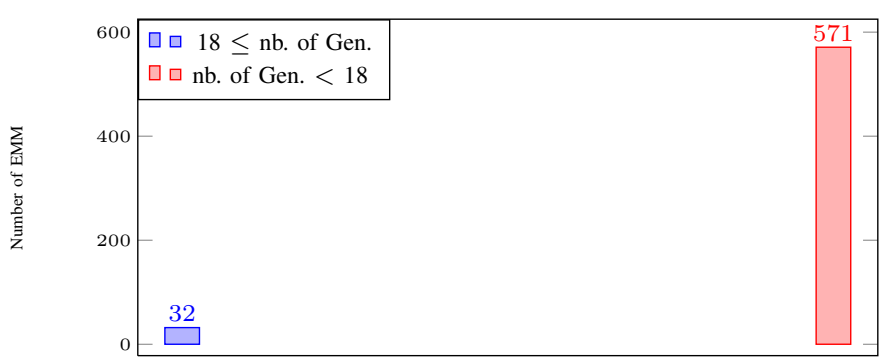

Fig. 6: Classification of modes with participation factor $\geq 10 \%$

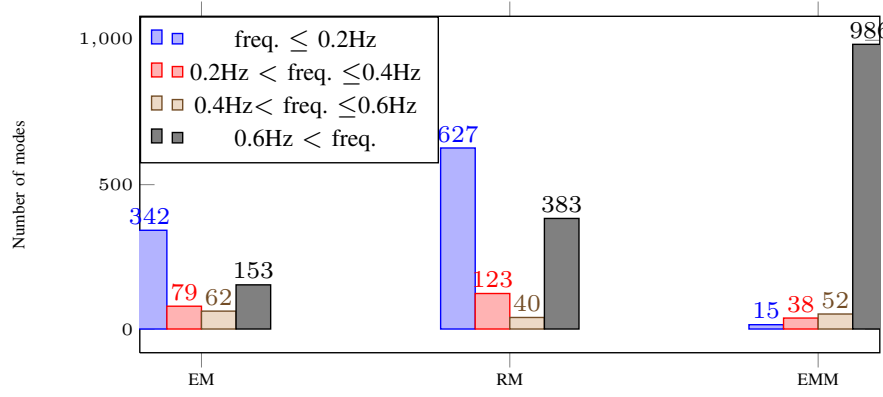

Fig. 7: Frequency classification

range ( $-\leq 0.2 H z-)$ in which these modes are considered). These modes are less spread (see also classification 3) and can thus be damped with more local control solutions as, for example, POD control of HVDC links.

4) Classification according to the damping: Classification according to the damping ratio is given in Fig. 8. Based on this classification more than half of the coupling modes are damped at more than $40 \%$ and thus do not have significant impact on system dynamics. Most of them are regulator and electrical modes but not electromechanical. Indeed, the critical (with damping less than 5\%) ones are of electromechanical nature.

\section{CONCLUSION}

In this paper, the results of full modal analysis of the European power system are presented. All coupling modes

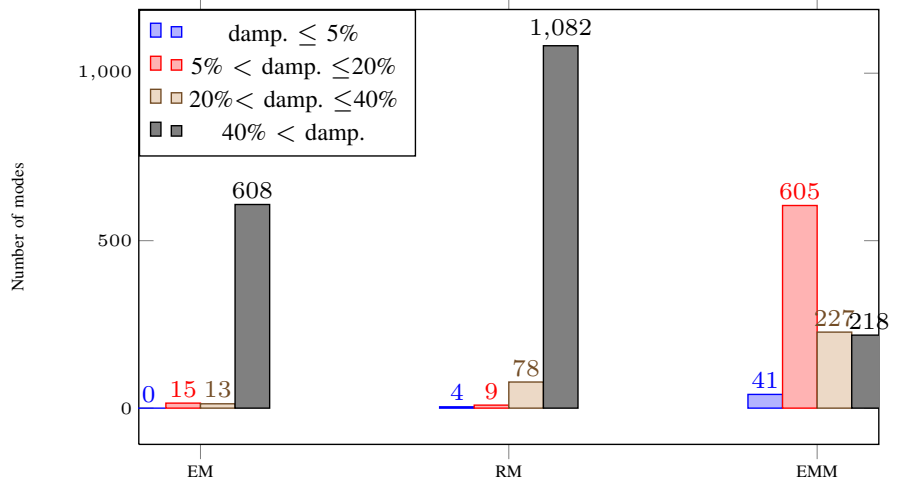

Fig. 8: Damping classification of the European power system are computed using a fully analytic method. The latter is exhaustive and does not require any a priori knowledge neither about the nature of modes nor about the participating devices. It was extended here to deal with large-scale systems.

For a given system, this kind of analysis allows one first to check the model: are any unstable modes? any abnormal frequencies of oscillations? Next, ranges of the dynamics of the system (slowest to fastest modes) are put into evidence. This is useful for control and model reduction actions. Finally, the couplings between the dynamic elements of the system are important to settle protection and control actions. The important novelty is to provide all this information in an exhaustive manner, i.e., to be able to make a general and full scan of the system. For the European system it is provided for the first time such exhaustive information. Dynamics are classified in accordance to their frequencies, spread of the participating devices, damping and nautre of the modes. This improved knowledge of the European power system will be exploited for any further dynamic correction actions: control, tuning of protection, defense plans,...

\section{REFERENCES}

[1] L. Arioua and B. Marinescu, Multivariable control with grid objectives of an HVDC link embedded in a large-scale AC grid, International Journal of Electrical Power \& Energy Systems, Vol. 72, pp. 99-108, 2015.

[2] L. Arioua and B. Marinescu, Robust grid-oriented control of high voltage DC links embedded in an AC transmission system, International Journal of Robust and Nonlinear Control, Vol. 26(9), pp. 1944-1961, 2016.

[3] J. Barquin, L. Rouco and H. R. Vargas, Generalized selective modal analysis, IEEE Power Engineering Society Winter Meeting, Vol. 2, pp. 1194-1199, 2002.

[4] M. Ellis, and P. D. Christofides, Selection of control configurations for economic model predictive control systems, AIChE Journal, Vol. 60, no. 9, pp.3230-3242, 2014.

[5] E. Grebe, et al. Low frequency oscillations in the interconnected system of continental Europe. In: IEEE PES General Meeting. IEEE, pp. 1-7, July 25-29, Providence, RI, USA, 2010.

[6] M. Kouki, B. Marinescu and F. Xavier, (2018), Eigencalculation of Coupling Modes in Large-Scale Interconnected Power Systems with High Power Electronics Penetration, 2018 Power Systems Computation Conference (PSCC), 1-7 June, Dublin, 2018.

[7] M. Kouki, B. Marinescu and F. Xavier, Exhaustive Modal Analysis of Large-Scale Interconnected Power Systems with High Power Electronics Penetration, IEEE Transactions on Power Systems, Vol. 35(4), pp. 2759$2768,2020$.

[8] I. Munteanu, B. Marinescu and F. Xavier (2017). Analysis of the interactions between close HVDC links inserted in an AC grid, In. Proc. IEEE Power \& Energy Society General Meeting, July 16-20, Chicago, Illinois, USA.

[9] L. Rouco, I.J. Pérez-Arriaga, R. Criado Calero and J. Soto, A computer package for analysis of small signal stability in large electric power systems, Proceedings of the $11^{\text {th }}$ Power Systems Computation Conference, pp. 1141-1148, Avignon (France), August 1993.

[10] X. Yin, and J. Liu, Input-output pairing accounting for both structure and strength in coupling, AIChE Journal, Vol. 63, no. 4, pp. 1226-1235, 2017.

[11] M. Luther, I. Biernaka and D. Preotescu, (2010), "Feasibility Aspects of a Synchrounous Coupling of the IPS/UPS with the UCTE", CIGRE Session, C1-204, Paris, France , 2010 